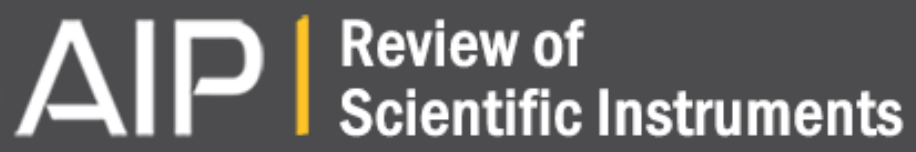

\section{A rotating directional probe for the measurements of fast ion losses and plasma rotation at Tokamak Experiment for Technology Oriented Research}

M. Rack, Y. Liang, H. Jaegers, J. Aßmann, G. Satheeswaran, Y. Xu, J. Pearson, Y. Yang, P. Denner, and L. Zeng

Citation: Review of Scientific Instruments 84, 083501 (2013); doi: 10.1063/1.4816821

View online: http://dx.doi.org/10.1063/1.4816821

View Table of Contents: http://scitation.aip.org/content/aip/journal/rsi/84/8?ver=pdfcov

Published by the AIP Publishing

\section{Articles you may be interested in}

Scanning ion sensitive probe for plasma profile measurements in the boundary of the Alcator C-Mod tokamak Rev. Sci. Instrum. 84, 053507 (2013); 10.1063/1.4807699

Inversion technique to obtain local rotation velocity and ion temperature from line-integrated measurements for elongated tokamak plasmaa)

Rev. Sci. Instrum. 83, 10D717 (2012); 10.1063/1.4733732

Characteristics of geodesic acoustic mode zonal flow and ambient turbulence at the edge of the HL-2A tokamak plasmas

Phys. Plasmas 14, 122301 (2007); 10.1063/1.2817047

Investigation of self-organized criticality behavior of edge plasma transport in Torus experiment of technology oriented research

Phys. Plasmas 11, 5413 (2004); 10.1063/1.1810160

Fast neutral lithium beam probing of the edge region of the spherical tokamak ETE

Rev. Sci. Instrum. 75, 3471 (2004); 10.1063/1.1784534

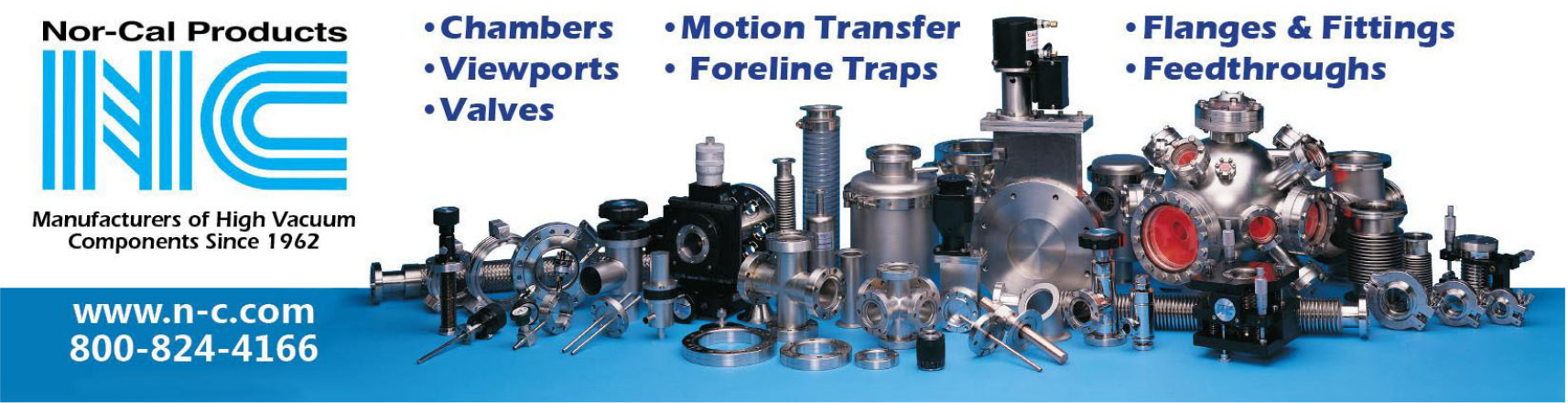




\title{
A rotating directional probe for the measurements of fast ion losses and plasma rotation at Tokamak Experiment for Technology Oriented Research
}

\author{
M. Rack, ${ }^{1}$ Y. Liang, ${ }^{1}$ H. Jaegers, ${ }^{1}$ J. Aßmann, ${ }^{1}$ G. Satheeswaran, ${ }^{1} \mathrm{Y} . \mathrm{Xu},{ }^{2}$ J. Pearson, ${ }^{1}$ \\ Y. Yang, ${ }^{3}$ P. Denner, ${ }^{1}$ and L. Zeng ${ }^{1,3}$ \\ ${ }^{1}$ Institute of Energy and Climate Research - Plasma Physics, Forschungszentrum Jülich GmbH, \\ Association EURATOM-FZJ, Partner in the Trilateral Euregio Cluster, D-52425 Jülich, Germany \\ ${ }^{2}$ Laboratoire de Physique des Plasmas - Laboratorium voor Plasmafysica, Association Euratom-Belgian state, \\ Ecole Royale Militaire - Koninklijke Militaire School, Partner in the Trilateral Euregio Cluster, \\ B-1000 Brussels, Belgium \\ ${ }^{3}$ Institute of Plasma Physics, Chinese Academy of Sciences, Hefei 230031, China
}

(Received 25 April 2013; accepted 11 July 2013; published online 1 August 2013)

\begin{abstract}
This work discusses a new directional probe designed for measurements of fast ion losses and the plasma rotation with a high angular resolution in magnetically confined plasmas. Directional and especially Mach probes are commonly used diagnostics for plasma flow measurements, and their applicability for the fast ion losses detection has been demonstrated. A limitation of static Mach probes is their low angular resolution. At the Tokamak Experiment for Technology Oriented Research, the angular resolution is strongly restricted by the finite number of available measurement channels. In a dynamic plasma, where instabilities can lead to local changes of the field line pitch-angle, plasma flow, or fast ion losses, a low angular resolution makes a precise data analysis difficult and reduces the quality of the measured data. The new probe design, the rotating directional probe, combines the features of early directional probes and Mach probes. It consists of two radially aligned arrays of nine Langmuir probe pins with each array facing opposite directions. During the measurement the probe head rotates along its axis to measure the ion saturation current from all directions. As a result, the rotating directional probe simultaneously provides an angular dependent plasma flow and fast ion losses measurement at different radial positions. Based on the angular dependent data, a precise determination of the current density is made. In addition, the simultaneous measurement of the ion saturation current at different radial positions allows for resolving radially varying field line pitch-angles and identifying the radial dynamic of processes like fast ion losses. [http://dx.doi.org/10.1063/1.4816821]
\end{abstract}

\section{INTRODUCTION}

Thermonuclear fusion is foreseen as one of the future energy resources. In the first 50 years of research, the main focus has been on axisymmetric magnetic confinement devices, for which the toroidal magnetic field is described as twodimensional, assuming toroidal symmetry. Current developments tend to make a three-dimensional treatment of the magnetic field necessary, e.g., in stellarators and tokamaks with edge localized mode (ELM) control via resonant magnetic perturbations (RMPs). ${ }^{1}$ The effects of such non-axisymmetric magnetic fields on the plasma and its confined particles are an open question. One of the key aspects is the fast ion confinement, since a sufficient $\alpha$-particle confinement is needed for an ignition of the fusion plasma; for the study of this, highly suited diagnostics are needed.

Directional and Mach probes are commonly applied diagnostics for plasma flow measurements in magnetically confined plasmas. ${ }^{2,3}$ Their usability for fast ion losses measurements has been shown. ${ }^{4}$ For both usages, the difference between probe signals measured from Langmuir probe pins facing opposite directions contains the information about the asymmetric distribution of plasma parameters, including the plasma rotation and fast ion losses.

Langmuir probes are a simple, well established plasma diagnostics measuring the main plasma parameters: electron temperature, density, and ion saturation current. They are used in various fields, apart from fusion, for the characterisation of magnetised and non-magnetised plasmas, e.g., space, medical, and industrial research.

The early directional probes, e.g., presented by Hudis and Lidsky, ${ }^{2}$ used one channel for such measurements and were rotated during the discharge through $180^{\circ}$ to measure the ion saturation current from both directions. This necessarily assumes that the plasma parameters are constant over a long time, i.e., before and after the rotation of the probe. More recent Mach probes ${ }^{5-7}$ consist of several Langmuir probe pins distributed around the probe head allowing for a measurement of the ion saturation current to the probe from different directions at the same time. Thus, an assumption of constant plasma parameters during the measurement is not necessary. In addition, an angular dependence of the ion saturation current can be detected, allowing for the simultaneous measurement of parallel and perpendicular plasma flows.

However, the angular resolution reached by these probes is low and often limited due to the number of available channels of the probe manipulator. Especially, at the Tokamak Experiment for Technology Oriented Research (TEXTOR), ${ }^{8}$ the number of channels for reciprocating probes is limited to 20 due to the technical specifications of the fast probe manipulator. ${ }^{9}$ Therefore, such a probe at TEXTOR can only 
give a very rough angular dependence. A technique to achieve a much higher angular resolution is a rotating probe as presented by Dyabilin et al. ${ }^{10}$ The rotating Mach probe, described therein, measures the parallel and perpendicular plasma rotation at one radial position. This allows for a very accurate determination of the Mach number, but only at one radial location. It misses the radial dependence, e.g., the radial correlation of physical quantities such as fast ion losses, ELM filaments, and turbulence.

This paper describes a rotating directional probe which achieves a high angular resolution and a simultaneous measurement of the ion saturation current at different radial positions. The latter is of particular importance for obtaining the radial profile of the plasma rotation and fast ion losses, as well as the radial correlation of these quantities. The probe combines the rotation of a directional probe and the contemporaneous measurement of the ion saturation current from opposite directions of a Mach probe. During the rotation an angular dependent ion saturation current is measured. A radial profile covering $4.8 \mathrm{~cm}$, with a spatial resolution of $6 \mathrm{~mm}$, is provided. In this article, the design of the rotating directional probe (Sec. II) and the experimental set-up used for the probe measurements at TEXTOR (Sec. III) are described. The measurement technique (Sec. IV) and first results (Sec. V) for the fast ion losses and plasma rotation measurement are presented. The advantages and limitations of the rotating directional probe in comparison with static Mach probes are discussed (Sec. VI).

\section{ROTATING DIRECTIONAL PROBE DESIGN}

One of the key points in the design of such a probe is the materials used. Two main requirements exist for the probe head of the rotating directional probe: (1) it has to sustain a high heat load of plasmas, (2) it has to be mechanically stable to withstand the linear and rotary acceleration. In addition, low-Z materials are preferred to decrease impurities released to the plasma. For a sufficient plasma rotation measurement half a rotation turn is needed. This means that at the maximum rotation speed available the probe head has to stay in the plasma for at least $129 \mathrm{~ms}$. Including the time taken to plunge to the measurement position the total plasma facing time will be $450 \mathrm{~ms}$. An obvious advantage, compared to standard Mach probes, is the rotation which distributes the heat load equity around the probe head.

Boron nitride (HeBoSint) has been chosen for the probe head material due to its high temperature resistance. Its mechanical stability has been tested to be strong enough for a linear movement with $0.3 \mathrm{~m} \mathrm{~s}^{-1}$ and a maximum rotation speed of $4 \mathrm{~Hz}$, so as not to harm the material. During the measurement of the plasma rotation, in an ohmic heated plasma, with the probe placed $3 \mathrm{~cm}$ inside the last closed flux surface (LCFS) an innocuous heat increase was noticed.

The probe head has a cylindrical shape with a crosssection diameter of $30 \mathrm{~mm}$ and a total length of $160 \mathrm{~mm}$. Its modular design contains 14 segments: nine equipped with two Langmuir probe pins and five without probe pins; see Fig. 1. All segments are conjoined with two stainless steel screws that are sealed with boron nitride caps on the plasma

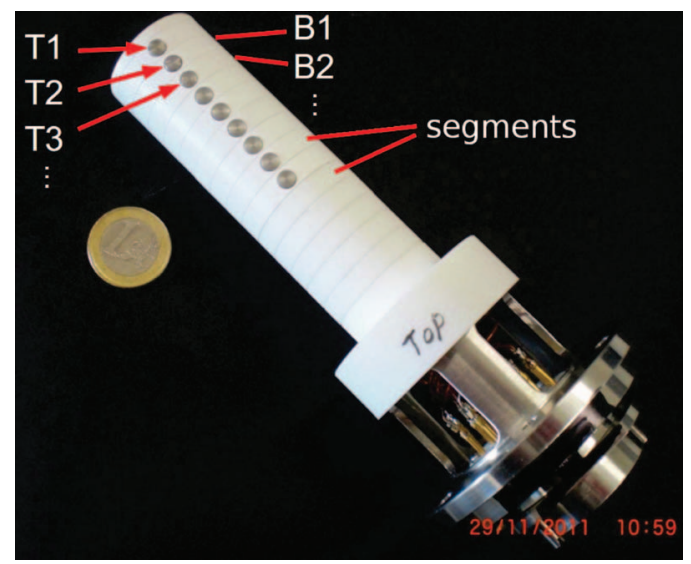

FIG. 1. Picture of the probe head. The probe consists of nine segments with two and five segments with no Langmuir probe pins. The Langmuir probe pins are labelled with $\mathrm{T} 1, \mathrm{~T} 2, \mathrm{~T} 3, \ldots$ (seen in the picture) and $\mathrm{B} 1, \mathrm{~B} 2, \ldots$ on the opposite side of the probe head.

facing side. The probe pins on the one side are labelled as $\mathrm{T} 1, \mathrm{~T} 2, \mathrm{~T} 3, \ldots$ and those facing in opposite directions as B1, $\mathrm{B} 2, \ldots$ The radial distance between neighbouring Langmuir probe pins is $6 \mathrm{~mm}$. Each probe pin is made out of tungsten and has a diameter of $4.55 \mathrm{~mm}$. The copper wires attached to the Langmuir probe pins are isolated with ceramic plates connecting to the 20 pin feed through at the base of the probe head, to fit to the TEXTOR probe manipulator.

The Langmuir probes are operated in the ion saturation regime; a biasing of $-176 \mathrm{~V}$, against the TEXTOR liner is applied. For this purpose a battery array is used to keep the noise level as low as possible. A typical noise level for the system is less than $4 \mathrm{~mA}$. The data acquisition uses a CT 1-T current transducer of the company LEM for the conversion of the measured currents to voltage signals and INCAA TR32 modules for the digitalisation of such. Data are acquired at $0.5 \mathrm{MHz}$ to provide an accurate angular resolution of the measurement. The linear and rotary motion of the fast reciprocating probe is detected by standard potentiometers recorded by computer-automated measurement and control (CAMAC) modules.

The rotating directional probe is operated in two modes: (a) for fast ion losses detection and (b) for plasma rotation measurement. The fast ion losses are detected with the probe positioned outside of the LCFS. In the rotation measurement mode, the probe is positioned in a range of $r=43 \mathrm{~cm}-48 \mathrm{~cm}$ in the plasma edge. A combination of both modes is possible.

\section{EXPERIMENTAL SET-UP}

TEXTOR is a medium size tokamak with a major radius of $1.75 \mathrm{~m}$ and a minor radius of about $48 \mathrm{~cm}$. It has a circular poloidal plasma cross-section. TEXTOR is equipped with neutral beam injection (NBI) and ion cyclotron resonance heating (ICRH) for an auxiliary plasma heating of 7 MW. The two NBIs are arranged tangentially, facing into opposite directions, allowing for independent control of the NBI heating and injection direction of fast particles, as it is used in our experiments to generate the fast ions. Its heating power is changed either via the aperture of the beam, which changes 
TEXTOR pulse 117939

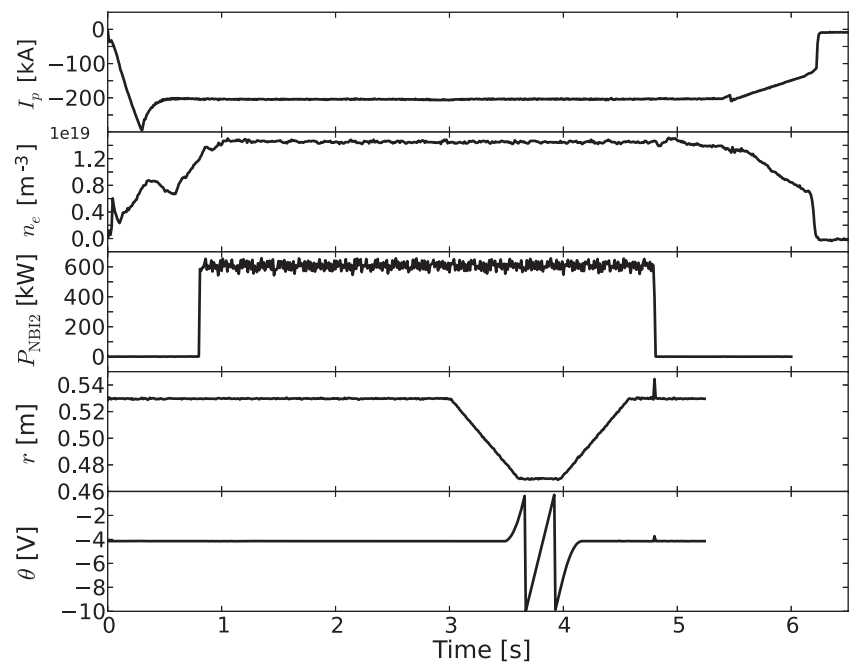

FIG. 2. Typical time-traces for a fast ion losses experiment with the rotating directional probe at TEXTOR. From top to bottom: the plasma current $\left(I_{p}\right)$, the electron density $\left(n_{e}\right)$, the power of the NBI $2\left(P_{\mathrm{NBI}}\right)$, the radial probe position $(r)$, and the rotation angle $(\theta)$.

the amount of injected neutrals, or by adjusting the acceleration speed, which influences the energy of the injected neutrals.

In the conducted experiments for fast ion losses and plasma rotation studies the typical plasma parameters at TEXTOR are core electron temperatures of $1.5 \mathrm{keV}$, and densities around $2 \times 10^{19} \mathrm{~m}^{-3}$ at plasma currents of about $200 \mathrm{kA}$, and magnetic fields of $2 \mathrm{~T}$. Figure 2 shows timetraces of a plasma discharge at TEXTOR with auxiliary NBI heating for inducing fast ions. In that discharge, the rotating directional probe is plunged to the position of minor radius $r$ $=47 \mathrm{~cm}$. The probe rotation angle, $\theta$, is given in the range of $-10 \mathrm{~V}$ to $0 \mathrm{~V}$, which corresponding to $0^{\circ}-360^{\circ}$. During this pulse the probe head is rotated by $720^{\circ}$, the first and last $180^{\circ}$ are needed for the acceleration/deceleration of the rotation, the measurement takes place during the centre turn.

The probe head of the rotating directional probe is designed for the usage on the fast reciprocating probe at TEXTOR. The fast reciprocating probe is placed on the midplane of the low field side. It can move probes linearly in the radial direction along the mid-plane and rotate them along the probes head major axis. The linear movement is limited from $r=53 \mathrm{~cm}$ to $39 \mathrm{~cm}$, at a speed of up to $5 \mathrm{~m} \mathrm{~s}^{-1}$; for the rotating directional probe a speed of $0.3 \mathrm{~m} \mathrm{~s}^{-1}$ is adequate. Its rotation reaches a frequency of around $4 \mathrm{~Hz}$.

\section{MEASUREMENT TECHNIQUES}

Each pair of Langmuir probe pins, mounted on opposite sides of one segment, measures the ion saturation currents in both co- $\left(j_{\mathrm{Co}}\right)$ and counter-current $\left(j_{\mathrm{Ctr}}\right)$ directions which are proportional to the parallel particle fluxes $\Gamma$, as sketched in Fig. 3.

Due to the rotation feature the ion saturation current detected on one Langmuir probe pin of the directional probe may provide the full information for both the co- and counter-

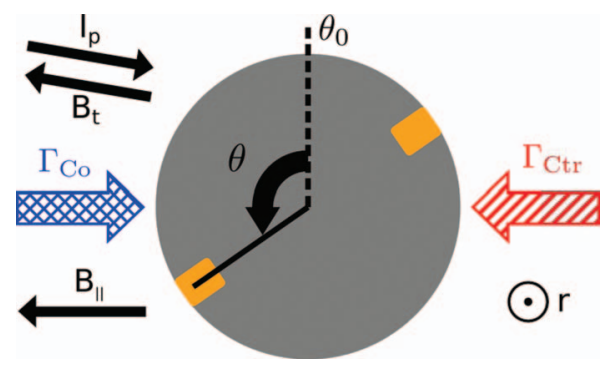

FIG. 3. Sketch of a segment of the rotating directional probe. One segment contains two Langmuir probe pins (orange). Both probe pins are facing into opposite directions to measure the co- and counter-going particle fluxes, $\Gamma_{\mathrm{Co}}$ and $\Gamma_{\mathrm{Ctr}}$, simultaneously. The probe head rotates anti-clockwise (looking towards the core of the device). $I_{p}$ and $B_{t}$ give the toroidal plasma current and toroidal magnetic field direction, $B_{\|}$indicates the direction of the parallel component of the magnetic field, and $r$ is the direction of the minor radius. $\theta$ is the rotation angle and $\theta_{0}$ is the reference angle when the probe pin areas are perpendicular to the parallel flux.

going particle fluxes (for constant plasma parameters during the rotation); see Hudis and Lidsky. ${ }^{2}$ Figure 4 shows the predicted signal of such a measurement, where $I_{\text {sat }}$ is the ion saturation current and $\theta$ is the rotation angle. With non-changing plasma parameters a constant particle flow in the scrape-off layer (SOL)/plasma edge during the measurement can be assumed. The maximum current from each direction is detected when the Langmuir probe pin area stands normal to the particle flux direction. For a non-zero plasma flow in an ohmic plasma the particle flux differs from the co- and countercurrent directions.

Furthermore, since the rotating directional probe is equipped with two Langmuir probe pins on each side only half a turn of the probe head is needed to measure the coand counter-current going particle fluxes. Consequently, this brings two advantages: first, the measurement time is halved, which reduces the total thermal load on the probe head, second, the probe measurement becomes less frail for changes in the main plasma parameters. Local pitch-angle changes do not disturb the measurement.

As a result of the rotation of the probe head and the fast data acquisition, a precise angle dependence of the ion saturation current, $I_{\text {sat }} \equiv I_{\text {sat }}(\theta)$, is known. From this the current density, $j$, as a representative for the particle flux $\Gamma \sim j$ can be calculated. $j$ can be substituted with either $j_{\text {Co }}$ or $j_{\text {Ctr }}$ depending on the angular position of the Langmuir probe pin that is

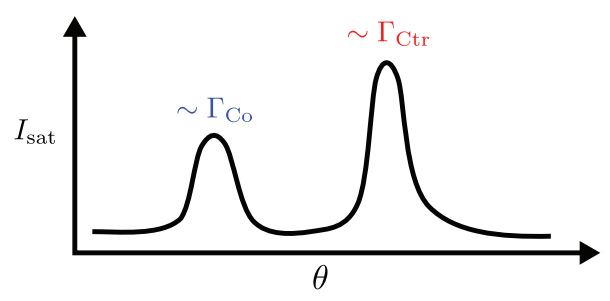

FIG. 4. Example for an ion saturation current measured by one Langmuir probe pin during the rotation of the probe head by $360^{\circ}$ with non-zero parallel flow. $I_{\mathrm{sat}}$ is the measured ion saturation current and $\theta$ is the rotation angle of the probe. The two maxima are proportional to the parallel particle flux in co$\left(\Gamma_{\mathrm{Co}}\right)$ and counter-current $\left(\Gamma_{\mathrm{Crt}}\right)$ directions. 
considered. For the current density $j$ we know

$$
j=\frac{I_{\mathrm{sat}}(\theta)}{A_{\mathrm{eff}}(\theta)},
$$

with $A_{\text {eff }}$, the effective area of the corresponding probe pin, and $I_{\text {sat }}$, the angle dependent ion saturation current measured from the co-/counter-side, respectively. The effective area can be written as $A_{\text {eff }}=A \sin \left(\theta-\theta_{0}\right)$, where $A$ is the probe pin area. $\theta_{0}$ gives the probe head angle at which $A$ is perpendicular to the particle flux. With a well aligned probe, regarding the geometrical axis, the local pitch-angle can be determined from $\theta_{0}$. Following Eq. (1), the current density can be found by applying a sine fit to the measured data:

$$
\frac{I_{\mathrm{sat}}(\theta)}{A}=j \sin \left(\theta-\theta_{0}\right),
$$

with the fitting parameters $j$ and $\theta_{0}$.

Figure 5 shows the measured data and the fitting results (solid line) for a pair of Langmuir probe pins at $r=45.2 \mathrm{~cm}$ in an ohmic heated plasma. A mean square algorithm is used for the fit. The curve fits about half the measured data in its maximum region $\left(45^{\circ}-135^{\circ}\right)$, but fails near $0^{\circ}$ and $180^{\circ}$, where higher saturation currents are measured than expected (cf. Refs. 10 and 11). This increase, when the normal of the probe pin area is perpendicular to the plasma flow direction, can be understood as "gyration hits" of passing ions. At that plasma region ions have a gyration radius of about $0.4 \mathrm{~mm}$ in ohmic TEXTOR plasmas. There guiding centre would pass the probe head, but due to their gyration they hit the Langmuir probe pins once it is in a perpendicular position to the plasma flow.

An approach to compensate the gyration hits and get more accurate values from the fit is an additional term in the fit function that takes perpendicular gyration hits into account.

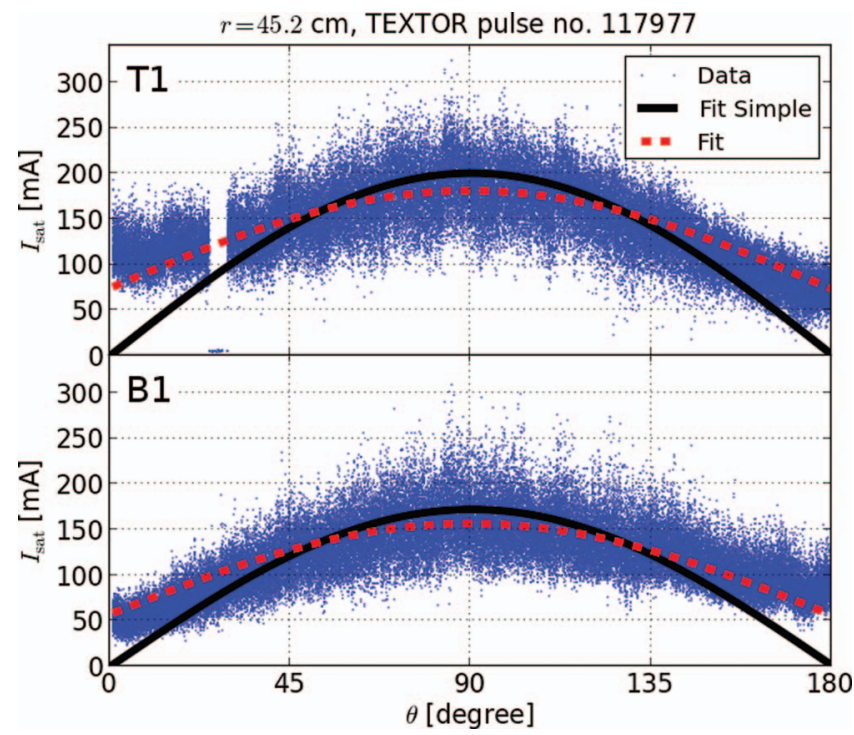

FIG. 5. Data of the rotating directional probe for two opposite facing Langmuir probe pins at $r=45.2 \mathrm{~cm}$ inside of the LCFS. The dots show the measurement, and the solid and dashed lines give the fitting curves. The dashed curve corrects for gyration hits of passing ions. The view is limited to half a turn of the probe head, since this contains the whole information on the plasma flow.
It extends Eq. (2) to

$$
\begin{aligned}
\frac{I_{\mathrm{sat}}(\theta)}{A}= & j \sin \left(\theta-\theta_{0}\right) \\
& +j_{\mathrm{gyr}}\left[1-\sin \left(\theta-\theta_{0}\right)\right],
\end{aligned}
$$

with $j_{\text {gyr }}$ representing the gyration hits. Similar to $j, j_{\text {gyr }}$ is handled separately for each Langmuir probe pin. The comparison in Fig. 5 presents a much better fit resulting from this extended fit function (dashed line).

The spontaneous drop in the measured signal of the Langmuir probe on top of Fig. 5 at $30^{\circ}$ originates from a technical trip of the electrical connection. Such a drop of the signal below the noise level does not effect the analysis as these regions are not taken into account for the fitting of the data.

The accurate determination of the current density from both sides, as described here, is the basis for the calculation of the fast ion losses and the plasma rotation. Discussions by Goubergen et al. ${ }^{12}$ pointed out that $j$ depends on the parallel and perpendicular flows $j \sim \exp \left\{v_{\|} / c_{s}-v_{\perp} \cot (\theta) / c_{s}-1\right\}$, where $v_{\|}$and $v_{\perp}$ are the parallel and perpendicular flow velocities and $c_{s}$ is the ion sound speed. Following this, a consideration of the perpendicular flow is necessary for the calculations of the current density. During the measurement in the SOL in the presence of fast ion losses the parallel flow is strongly enhanced by the ion losses and becomes dominant over the perpendicular, $v_{\|} \gg v_{\perp}$ (see also discussion in Sec. IV A). Therefore, the perpendicular flow can be neglected and no $\theta$ dependence of $j$ needs to be considered. However, during measurements in the edge of an ohmic plasma large perpendicular flows may appear that cannot be neglected. The application of the presented analysis technique is then strongly restricted to a $\theta$-range $\left(80^{\circ}<\theta-\theta_{0}<100^{\circ}\right)$ where the $v_{\perp}$-term becomes negligibly small. For determining the perpendicular flow, the method described in Refs. 10-12 needs to be applied which will not be further discussed here. Instead the main focus is on fast ion losses measurements.

With the knowledge of the current density from opposite facing probe pins the two physical quantities fast ion losses and plasma rotation can be calculated by taking the difference or ratio of the current densities, respectively. The fast ion losses measurements are performed with the probe head located in the SOL whereas the plasma rotation measurements take place in the plasma edge and SOL.

\section{A. Fast ion losses measurement in the SOL}

Nagaoka et al. presented the general applicability of a directional probe for the measurement of fast ion losses. ${ }^{4}$ Fast ions are generated with a tangential NBI for the performed experiments. The NBI will cause, on the one hand, fast ion losses and on the other hand, a change in the plasma rotation. In the SOL, the asymmetry caused by the fast ion losses is dominant over that caused by the plasma rotation, as sketched in Fig. 6(a), and therefore the asymmetry of the velocity distribution of the bulk plasma can be neglected in the analysis.

The hatched region in Fig. 6(a) corresponds to the amount of particles flowing in counter-current direction that are detected by one Langmuir probe pin and the cross-hatched 
(a)
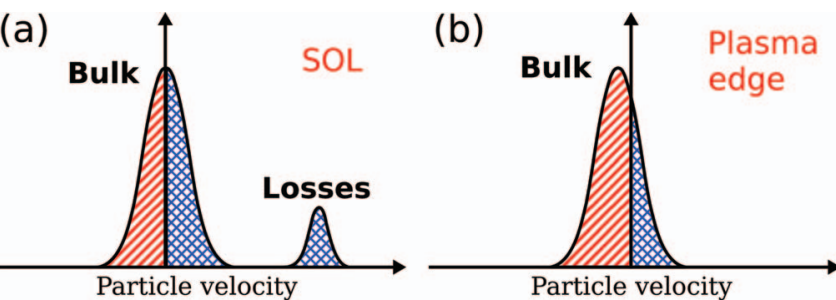

FIG. 6. Sketch of (a) the velocity distributions in the SOL of a beam heated plasma with a dominant fast ion losses contribution and (b) the velocity distribution in the edge of a counter rotating ohmic plasma. The hatched and cross-hatched regions correspond to the particle amount flowing in counterand co-current direction, respectively. See Fig. 3 for further information on the flow directions with respect to the probe head geometry.

region corresponds to the co-going particles detected by the probe pin facing in the opposite direction (see Fig. 3 for a better visualisation of the flow direction with respect to the probe head). In a beam heated plasma, $j_{\mathrm{Ctr}}$ is the current density from the bulk ions and $j_{\text {Co }}$ contains, in addition, a large fraction of the beam induced fast ion losses. The current density resulting from the fast ion losses, $j_{\text {loss }}$, can be evaluated by taking the difference of $j_{\mathrm{Co}}$ and $j_{\mathrm{Ctr}}$ :

$$
\begin{aligned}
& j_{\text {Co }}=j_{\text {Bulk }}+j_{\text {loss }}, \\
& j_{\text {Ctr }}=j_{\text {Bulk }} .
\end{aligned}
$$

It follows for a beam heated plasma

$$
j_{\text {loss }}=j_{\mathrm{Co}}-j_{\mathrm{Ctr}},
$$

as a measure for fast ion losses in the SOL.

\section{B. Plasma rotation measurement}

In an ohmic plasma, the rotating directional probe can be used to measure the local plasma rotation. Figure 6(b) shows a sketch of the velocity distribution in the edge of a counter rotating plasma. It gives the general idea on the background of the measurement.

From the ratio of the ion saturation current measured in co- and counter-directions the local Mach number, $M$, can be determined as a measure for the plasma rotation

$$
M=k \ln \left(\frac{j_{\mathrm{Co}}}{j_{\mathrm{Ctr}}}\right),
$$

with $k$ a calibration constant taken as $k \approx 0.4$ based on the calculations for magnetised plasmas by Hutchinson. ${ }^{13} k$ depends on various plasma parameters, such as magnetic flux density and ion temperature, ${ }^{14}$ and cannot be calculated analytically for our cases in magnetised plasmas.

\section{EXPERIMENTAL RESULTS}

Figure 7 shows the fast ion losses measurement in a cobeam heated plasma for four TEXTOR pulses with reversed plasma current in the range $-180 \mathrm{kA}$ to $-285 \mathrm{kA}$. A reversed toroidal magnetic field of $-2.25 \mathrm{~T}$ at an electron density of $1.5 \times 10^{19} \mathrm{~m}^{-3}$ has been used and $600 \mathrm{~kW}$ hydrogen NBI heating was applied at an acceleration voltage of $U=50 \mathrm{kV}$.

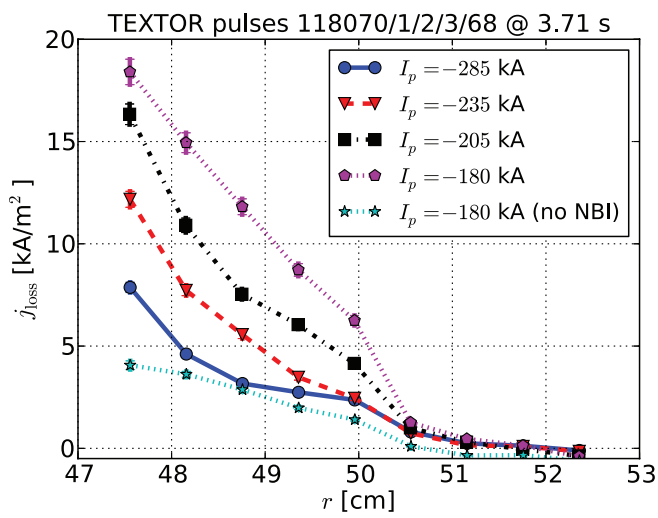

FIG. 7. Measured fast ion losses in a co-beam heated plasma. The dependence of fast ion losses on the plasma current is shown. A reference without beam induced fast ions is shown by the stars.

The observed plasma current dependence is in line with the known dependence of the critical loss energy of fast ions on the plasma current: ${ }^{15} E_{\text {loss }} \sim I_{p}^{2}$. It can easily be understood by considering the reduced confinement at lower plasma currents. In addition, a clear radial decay of the fast ion losses can be observed for all cases with vanishing losses at around $r=50.6 \mathrm{~cm}$. No losses can be detected further outside in this plasma configuration due to plasma facing components intercepting the fast ions.

In Fig. 8, the measurement of the plasma rotation, given as the Mach number, is shown. A slow counter rotation, $M \sim-0.1$, in the range of around $r \leq 46.3 \mathrm{~cm}$ is found which then turns to a co-rotation and increases towards $M \sim 0.8$ at the LCFS. In the SOL, a radial decay of the plasma rotation can be observed. Similar trends are seen in other TEXTOR plasmas as discussed by Xu et al. ${ }^{16}$ The higher absolute values seen in the presented discharge (Fig. 8) result from a different plasma configuration, here reversed plasma current and toroidal field are used compared to standard TEXTOR discharges.

The denoted error bars result from the inaccuracy of the Langmuir probe pin area, which contains an error of about $5 \%$, and the fit inaccuracy, usually around $1 \%$. Inaccuracies of the data acquisition are below $1 \%$ and therefore not considered.

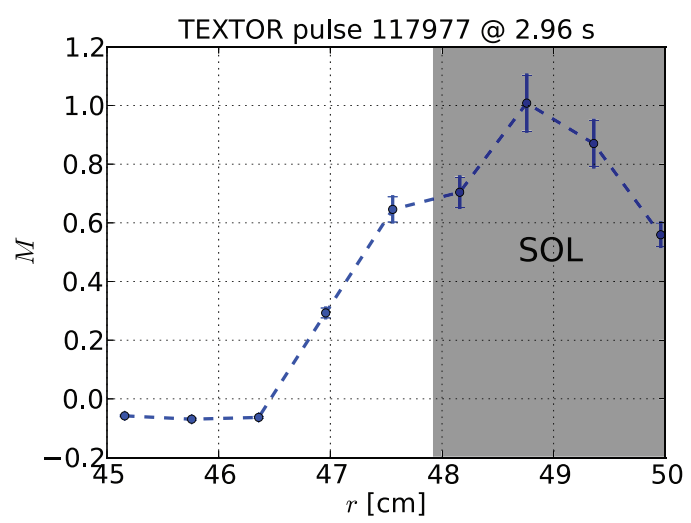

FIG. 8. Plasma edge and SOL Mach number measured in an ohmic plasma. 
Cross-correlation for $r=47.6 \mathrm{~cm}$, TEXTOR pulse no. 118068

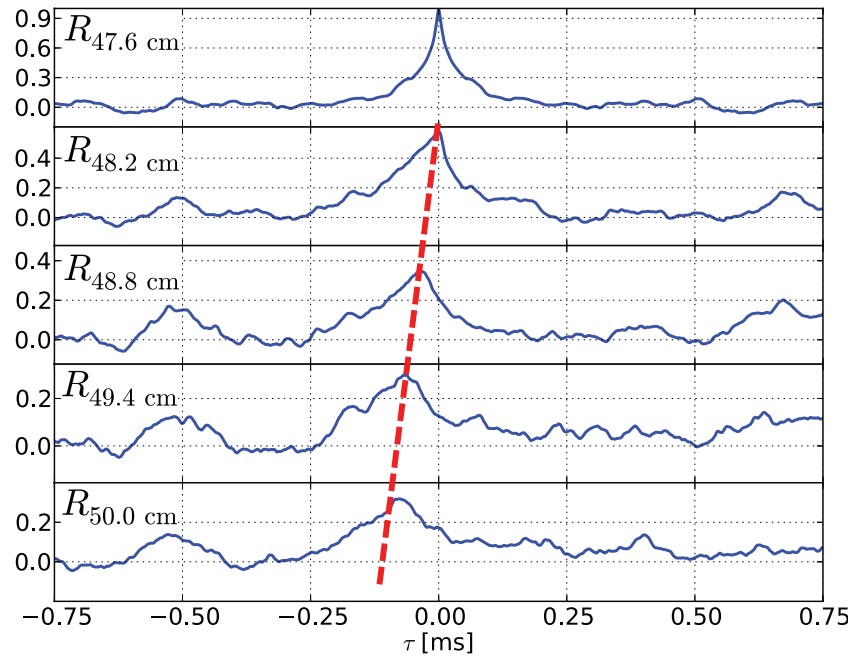

FIG. 9. Cross-correlation between the signal measured at $r=47.6 \mathrm{~cm}$ with signals measured further away from the plasma. The cross-correlations indicates the radial outward propagation of ion losses with a speed of about $200 \mathrm{~m} \mathrm{~s}^{-1}$ (marked by dashed line).

In addition to the fast ion losses and Mach numbers, the presented multi-pin directional probe may also provide further information about the radially dynamic of the plasma seen as high frequent modulations in the ion saturation current signal (cf. Fig. 5). These modulations can be found inside, as well as outside the LCFS. Fast changes in the ion saturation current can be caused by turbulences and magnetohydrodynamic instabilities such as saw teeth, tearing modes, and ELMs. Figure 9 depicts the radial cross-correlation of the Langmuir probe pin signal at $r=47.6 \mathrm{~cm}$ with those measured by further outside probe pins. The presented TEXTOR pulse 118068 has the probe set-up for the fast ion losses measurement, i.e., probe head in the SOL.

The cross-correlation is calculated based on

$$
R_{x y}(\tau)=\frac{1}{T} \int_{-T / 2}^{T / 2} x(t) \cdot y(t+\tau) \mathrm{d} t,
$$

where $x$ and $y$ are the measured signals at different radial positions, and $T$ is the considered time window for the correlation. The variable $\tau$ gives the lag between the signals.

For the given pulse, the cross-correlations allow us to determine the radial outward propagation of ion losses with a speed of around $200 \mathrm{~m} \mathrm{~s}^{-1}$ starting at a radial position between $r=48.2 \mathrm{~cm}$ and $48.8 \mathrm{~cm}$. The analysis of the radial correlations has been widely used in the study of edge turbulence by Langmuir probes ${ }^{17}$ and is helpful in exploring the radial dynamic of other processes, e.g., in the characterisation of ELM filaments where the presented probe system can be applied as an additional tool to visual observation techniques. ${ }^{18}$ In addition, the radial correlation is a useful tool to gain the phase information of externally applied dynamic RMPs ${ }^{19}$ which is a major issue in the understanding of RMP physics and has been found to have a radial dependence. $^{20}$

\section{DISCUSSION AND CONCLUSION}

The rotating directional probe is a suitable diagnostics for measurements in a non-axisymmetric dynamic magnetic field, e.g., with the applied dynamic ergodic divertor at TEXTOR. This is a strong advantage compared with earlier directional/Mach probes. The high angular resolution enables for a precise analysis of the ion saturation current, even in presence of dynamic perturbations. A precise adjustment of the probe head angle beforehand, as needed in the case of non-rotating probes to bring the probe pins in line with the parallel or perpendicular particle flow direction, is not necessary. The rotation of the probe head allows the whole angular dependence to be measured. Consequently, the maximum ion saturation current can be detected and, therefore, the fast ion losses and plasma rotation calculated. An angular misalignment or a local change of the pitch-angle does not perturb the measurements. Furthermore, radial changes in the pitchangle, e.g., due to non-axisymmetric fields, can be detected. As the probe consists of an array with nine probe pins it measures a $4.8 \mathrm{~cm}$ radial profile of fast ion losses in the SOL and the plasma rotation. Compared to a static Mach probe or the rotating Mach probe, the measurement of the rotating directional probe takes place at different radial positions simultaneously, whereas for a standard Mach probe it can provide a higher resolved radial dependence during one plunge, but misses radial correlation of the signals from different radii. Due to the design of the rotating directional probe, the radial resolution of the measured profiles is $6 \mathrm{~mm}$. To increase the radial resolution, the measurement is repeated in the same target plasma, shifting the probe by $1 \mathrm{~mm}$ each time. Thus a resolution of $1 \mathrm{~mm}$ is achieved; higher resolutions are unobtainable due to the limitations of the probe manipulator. This discomfort, compared to standard Mach probes, is a consequence of the probe design focussing on a high angular resolution.

The large radial range that can be covered by the rotating directional probe simultaneously is a strong achievement especially for the fast ion losses measurement. Previous results already showed the fast ion losses measurement with a directional probe at one radial position, but a radial dependent measurement of the fast ion losses could not be presented before. The precise knowledge of the radial dependence of fast ion losses helps for a further understanding of the processes responsible for the losses. Furthermore, the availability of simultaneously measured signals from different radial positions allows for estimating the radial cross-correlation of ion loss signals. The radial dynamic is valuable information for the investigation of the involved processes and becomes even more important in the presence of dynamic perturbations of the plasma.

Typical signal intensities of the rotating directional probe are in the order of a $100 \mathrm{~mA}$ with a signal to noise ratio below $4 \%$. The sub-structures in Fig. 5 with $\pm 50 \mathrm{~mA}$ on top of the main signal are caused by fast intermittent events such as turbulences that can be further characterised using the presented probe, which is not in the scope of this publication.

Due to the good signal to noise ratio and the high timeresolution of $0.5 \mathrm{MHz}$, the rotating directional probe is also 
well suited for studying ELM filaments, and the effect of RMPs on those. The typical ELM frequencies measured at TEXTOR are in the range of several hundred hertz and the maximum RMP rotation frequency is $5 \mathrm{kHz}$. In ELM filament studies during a limiter high-confinement mode (H-mode) discharge the rotating directional probe measurements focus on the filament propagation through the SOL, thus the probe is positioned outside of the LCFS. Due to the strongly increased heat fluxes in the confined plasma a safe operation of the probe inside the plasma cannot be guaranteed during a limiter $\mathrm{H}$-mode. With the probe outside of the plasma, the limiter takes the ELM heat loads and a safe operation of the probe is gained.

The usage of the rotating directional probe on the fast reciprocating probe at TEXTOR brings limitations for the presented probe which could be overcome by using an optimized probe manipulator. These limitations are: the maximum rotation speed of about $4 \mathrm{~Hz}$, and the limit of 20 available channels for measurement signals. A faster actuator for the rotation will reduce the time needed for the probe to stay in the plasma at a fixed radial position. If there was a larger number of available measurement channels, the probe head could be equipped with further arrays of Langmuir probe pins positioned at different angles, which could be described as a rotating Gundestrup probe. Such an improvement also reduces the waiting time of the probe during the rotation. However, more Langmuir probe pins will bring difficulties in the construction of the probe head. Due to the limitations and technical difficulties outlined these improvements are not seen to be advantages for the measurements currently performed with the rotating directional probe at TEXTOR.

The presented rotating directional probe, allowing for a highly resolved, angular dependent measurement of the ion saturation current, is well suited for the measurements of fast ion losses and plasma rotation in the presence of local pitch-angle changes and dynamic perturbations. Its highly angular resolved data and their radial correlation strongly extend the achievable information on fast ion losses and the plasma rotation. The main focus for the future work with the probe is the effect of RMP on the fast ion losses and plasma rotation.

\section{ACKNOWLEDGMENTS}

The help of the whole TEXTOR Team is gratefully acknowledged, with special thanks to Jörg Thomas, Sebastian
Kraus, D. Brinkmann, U. Schaufert, G. Esser, and D. Nicolai. Furthermore, M.R. thanks Ch. Perez von Thun for the valuable discussions. Support from the Helmholtz Association in frame of the Helmholtz-University Young Investigators Group VH-NG-410 is gratefully acknowledged.

${ }^{1}$ Y. Liang, "Overview of edge-localized mode control in tokamak plasmas," Fusion Sci. Technol. 59(3), 586 (2011).

${ }^{2}$ M. Hudis and L. M. Lidsky, "Directional Langmuir probe," J. Appl. Phys. 41(12), 5011 (1970).

${ }^{3}$ K.-S. Chung, "Mach probes," Plasma Sources Sci. Technol. 21(6), 063001 (2012).

${ }^{4}$ K. Nagaoka, M. Isobe, K. Shinohara et al., "Energetic ion measurements using a directional probe," Plasma Fusion Res. 1, 005 (2006).

${ }^{5}$ C. S. MacLatchy, C. Boucher, D. A. Poirier et al., "Gundestrup: A Langmuir/Mach probe array for measuring flows in the scrape-off layer of TdeV," Rev. Sci. Instrum. 63(8), 3923 (1992).

${ }^{6} \mathrm{~S}$. Jachmich, P. Peleman, M. Van Schoor et al., "First Mach probe measurements of rotation, electric field and particle transport in the DED-ergodized edge plasma of TEXTOR," in 33rd EPS Conference on Plasma Physics, Roma (Italy), 19-23 June 2006.

${ }^{7}$ X. Zhang, D. Dandurand, T. Gray et al., "Calibrated cylindrical Mach probe in a plasma wind tunnel,” Rev. Sci. Instrum. 82(3), 033510 (2011).

${ }^{8}$ O. Neubauer, G. Czymek, B. Giesen et al., "Design features of the tokamak TEXTOR," Fusion Sci. Technol. 47(2), 76 (2005).

${ }^{9} \mathrm{M}$. Mitri, "Control of a fast reciprocating probe for the TEXTOR fusion experiment," M.S. thesis (University Duisburg-Essen, 2005).

${ }^{10}$ K. Dyabilin, M. Hron, J. Stöckel et al., "Rotating Mach probe for ion flow measurements on the CASTOR tokamak," Contrib. Plasma Phys. 42(1), 99 (2002).

${ }^{11}$ J. P. Gunn, C. Boucher, P. Devynck et al., "Edge flow measurements with Gundestrup probes," Phys. Plasmas 8(5), 1995 (2001).

${ }^{12}$ H. V. Goubergen, R. R. Weynants, S. Jachmich et al., "A 1D fluid model for the measurement of perpendicular flow in strongly magnetized plasmas," Plasma Phys. Controlled Fusion 41(6), L17 (1999).

${ }^{13}$ I. H. Hutchinson, "Ion collection by probes in strong magnetic fields with plasma flow," Phys. Rev. A 37, 4358 (1988).

${ }^{14} \mathrm{~K} . \mathrm{S}$. Chung, "Why is the Mach probe formula expressed as $R=J_{\text {up }} /$ $J_{\mathrm{dn}}=\exp \left[K M_{\infty}\right]$ ?,” Jpn. J. Appl. Phys. 45(10A), 7914 (2006).

${ }^{15}$ W. Heidbrink and G. Sadler, "The behaviour of fast ions in tokamak experiments," Nucl. Fusion 34(4), 535 (1994).

${ }^{16}$ Y. Xu, C. Hidalgo, I. Shesterikov et al., "Role of symmetry-breaking induced by $E_{r} \times B$ shear flows on developing residual stresses and intrinsic rotation in the TEXTOR tokamak," Nucl. Fusion 53, 072001 (2013).

${ }^{17}$ Y. H. Xu, S. Jachmich, R. R. Weynants et al., "Investigation of selforganized criticality behavior of edge plasma transport in Torus experiment of technology oriented research," Phys. Plasmas 11(12), 5413 (2004).

${ }^{18}$ A. Kirk, B. Koch, R. Scannell et al., "Evolution of filament structures during edge-localized modes in the MAST tokamak," Phys. Rev. Lett. 96, 185001 (2006).

${ }^{19}$ O. Neubauer, G. Czymek, K. Finken et al., "The dynamic ergodic divertor in TEXTOR-A novel tool for studying magnetic perturbation field effects," in Proceedings of the 23rd Symposium of Fusion Technology [Fusion Eng. Design 75-79, 495 (2005)].

${ }^{20}$ Y. Yang, Y. Liang, Y. Sun et al., "Experimental observations of plasma edge magnetic field response to resonant magnetic perturbation on the TEXTOR tokamak," Nucl. Fusion 52(7), 074014 (2012). 